father. The ice, digitalis, and ether were continued. At lexcept one drachm of syrup of iodide of iron The

12 noon the patient had another very bal attack and a fatal result was expected, the temperature rising; but during the afternoon and evening she rallied and had several slightly lucid intervals. On the 14th she had had a much better night with fairly quiet sleep and nourishment was taken. The pupils slightly responded to light, which they had failed to do on the night of the 11th. During the last three days I had noticed that the temperature went up before the bowels acted and came down after the bowels had been relieved. During the next two days her condition was much the same. The attacks of coma were not so severe, and she took nourishment well. On the 17 th her condition was much the same. The bowels were kept acting freely. The patient could talk and answer questions in a whisper. The limbs were absolutely rigid and the toes were extended. The patient now complained of pain in both legs. The legs were gently rubbed for the first time. There were no patellar reflexes or ankle clonus. She was able to move her arms a little better, and the muscles of the neck were a little less rigid. She could also move her head a little. The pupils responded sluggishly to light. The muscles of the lower limbs had only wasted a little, and there was no marked emaciation. On the 18th the patient had had a good night. The temperature varied from $100^{\circ}$ to $1006^{\circ}$ and the pulse from 100 to 110 . The plantar reflexes were slightly present and she could just move her toes. The pupil reflexes were not so good. The general condition was much the same. On the 19 th the general condition was not so good, the patient seeming to be generally weaker. The symptoms were the same as on the previous day. At the middle of the day $I$ decided to give her iodide of potassium and solution of perchloride of mercury, alternating with quinine, iron, and digitalis. During the day the patient was very drowsy. The temperature rose to $102^{\circ}$ and the pulse became very rapid. Some cough developed during the day. The quantity of nourishment was increased. At 9 P.M. her condition was bad and I had her spine packed in ice. After ice had been applied she gradually improved and had had a fairly good night on the 20th. She complained during the night of pain in both legs. Ice was constantly applied and her temperature at 7 A.M. was $97.2^{\circ}$, the pulse being 80 and respiration good. At $730 \mathrm{~A} \mathrm{M}$. the ice was removed from the spire. During the night the patient had expressed a desire to have the bowels opened, and at 12 noon they acted. At 5.15 P.M. she passed urine consciously, and at 6.30 P.M. the bowels acted well. During the day ice was applied at intervals to the lumbar spine.

On May 21st the patient passed a fairly good night. The nurse informed me that she had been more conscious on passing frees but had taken no notice on passing urine. At 11.30 P.M. she asked for the bed-pan and used it, but urine passed involuntarily all day. On the next day there was no change, but at 8 P.M. the temperature rose to $101^{\circ}$; ice was immediately applied to the back. Her condition on the 23rd was much the same. Sbe had not had a good night, but when she was washed in the morning she was able to bend her knees very slightly. It was noticed that during the previous three or four days the patient had decidedly lost flesh. Daring the night and the day she had a troublesome cough. The temperature was $100^{\circ}$. There was no material change in her state on the next day. On the 25th she had passed a good night and could move her right leg slightly, but not the left. Her intellect seemed clearer. She passed a good night on the 26th taking nourishment freely. Her legs were rubbed twice a day. I saw Dr. Mitchell Bruce in London and discussed the case with him; we were of opinion that it was now one of myelitis of the dorsal region of the cord. It was, therefore, decided to increase the iodide and the solution of perchloride of mercury and to continue the massage. On the 27 th more movement in the lower limbs was noted. It was very slow but there was no pain. For the next three days the patient generally improved. On the 31 st she commenced taking solid food. On June 1st slight patellar reflexes were found to be present for the first time, and there was more power generally. The next day the improvement was marked and the sensation in the limbs was normal. The patient's condition had slowly improved on the 6th. The patellar reflexes were more marked. On the 11 th she had a sleepless night and was moved into another room. On the 28 th she was found to have steadily improved; she could walk from room to room and upstairs and downstairs. All medicine was stopped menstrual period came on on the 22nd. The patient's sight was distinctly affected, there being short sight for all small objects, also red to green colour-blindness. Most greens looked at first-i.e., two weeks previously, when this condition was first noticed-orange ; they now looked greener. Red and purples appeared as sage-green, green, or shades of mauve. She went out for a drive and she bad been in the garden for the last 10 days. On Sept 1st I learned that she had been away to the seaside. The patient was now perfectly well in every respect.

This case appears to me to be one of peculiar interest, as although spinal cord lesions occurring in the exanthus stage of measles are recorded they are nevertheless somewhat rare, and in a number of cases they have proved rapidly fatal. A case is recorded by Barlow $^{1}$ which presented a somewbat similar train of symptoms. In Barlow's case death occurred on the eleventh day. A detailed account of the post-mortem and microscopical appearances of the spinal cord are given at length and are most interesting. In my own case I would suggest that the changes in the cord were vascular in character and of greatest intensity in the dorsal region. Probably the vessels were surrounded by a zone of coagulated exudation and the neighbouring tissues were infiltrated with leucocytes. It is impossible to say whether there were any interstitial hæmorrhages. I think there can be no doubt that the bladder symptoms, the paralysis of the lower limbs, the degree of paresis which existed in the upper limbs, and the absence of knee-jerks and of plantar reflexes, were all spinal symptoms.

The question is, What was the relation between the myelitis and the measles? This patient was absolutely well before she sickened for measles. She contracted measles from a relative (who sat next to her at lunch and who developed a profuse rash on the next day). The incubation period was normal and a few days afterwards she had symptoms of meningitis, followed by symptoms of myelitis. I think that there are a sufficient number of cases on record to show that there is a distinct relation between measles and a form of myelitis which may be produced by the virus concerned in the other phenomena of measles.

I am very glad that I was able to have the opinions of Dr. Hughlings Jackson and Dr. Mitchell Bruce. At the time when they saw the case it was impossible to form any other opinion than that death must very shortly take place. The result of this case teaches us how in an apparently obviously hopeless condition a victory may be gained. Except for the incessant watching on the part of the nurses the patient would have died. On several occasions the pulse was uncountable and scarcely perceptible and I hardly thought that death could be warded off. It was interesting to note the comparatively rapid recovery, and I am sure that the iodide of potassium and solution of perchloride of mercury were of great use.

I have published this case as it may be of interest to practitioners, and it is comforting to know how absolutely complete recovery can occur in cases of this sort. I am greatly indebted to my partner, Mr. R. F. Chance, for his valuable aid and assistance during this very trying case.

Southampton.

\section{AN ANOMALOUS CASE OF CONTINUED FEVER WITH ABSCESSES OF THE} LIVER.

BY J. G. MoNAUGHT, M.A., M.D. GLasG., CAPTAIN, ROYAL ARMY MEDICAL CORPs.

THE following case may be of interest in connexion with that reported in THE LANCET of July 30th, 1898 (page 252), by Dr. Sidney Herbert Snell.

A gunner in the Royal Artillery, aged 24 years, was admitted to the special wards of the Station Hospital, Quetta, on Jan. 9th, 1898. For some time he had been suffering from intermittent fever, apparently of malarial origin. For two days at least before admission fever had been continuous. His temperature on admission was $102.6^{\circ} \mathrm{F}$. His face was flushed and his tongue was slightly furred. There was no abdominal distension or pain; he

I Transactions of the Royal Medical and Chirurgical Society, vol. lii. 
had three or four loose motions daily. The spleen was slightly enlarged; the heart and lungs appeared to be normal. On Jan. 12th his temperature was $100.4^{\circ}$ in the morning and $105^{\circ}$ in the evening; on the 13 th it was $100.4^{\circ}$ in the morning and $101 \cdot 6^{\circ}$ in the evening. On the 14 th he complained of severe pain in the right side aggravated by movement and by taking a deep breath. A friction rub was heard at the inferior angle of the right scapula on auscultation. There was no dulness on percussing the base of the lung. The lower border of the liver was tender on palpation. On the previous day he had had six loose, light yellow motions. On this date he passed about one ounce of bright blood from the bowel. The tongue was dry and brown in the centre. His blood gave incomplete reaction to Widal's test; distinct clumping occurred, but many bacilli remained actively motile at the end of half an hour. His temperature was $101.2^{\circ}$ in the morning and $100^{\circ}$ in the evening. On the 15th he seemed much better; the pain in the side was relieved, there was no diarrhoea, and the tongue was cleaner and moist. His temperature was $102.2^{\circ}$ in the morning and $103.8^{\circ}$ in the evening. On the 16 th there was a second hæmorrhage from the bowel, about five ounces of bright blood being passed. There was still pain in the right side on coughing, the respiratory murmur was very feeble, and the percussion note over the extreme base posteriorly was dull. The facies was now very suggestive of enteric fever, the tongue was brown and dry in the centre, and red at the tip and edges. The abdomen was somewhat distended. There were no rose spots. The blood gave a marked but incomplete reaction to Widal's test. The blood was examined for malarial parasites, but only one degene. rated pigmented spherical body was found. His temperature was $100^{\circ}$ in the morning and $102^{\circ}$ in the evening. On the 17 th he was restless and had passed a bad night. His temperature was $101.6^{\circ}$ in the morning and $103^{\circ}$ in the evening. His pulse was 140 and his respirations were 48 per minute. His pulse was very weak; his tongue was brown in the centre, furred at the sides, and red at the tip and edges; his abdomen was more distended and there were no rose spots; a small motion was passed containing some dark altered blood and some bright blood. On the 18th his condition improved his temperature was $100.4^{\circ}$ in the morning and $104.6^{\circ}$ in the evening. On the 19th he complained of abdominal pain and was perceptibly weaker; one motion was passed containing altere blood; his temperature was $100^{\circ}$ in the morning and $102 \cdot 6^{\circ}$ in the evening. On the 20 th hia condition was somewhat better. He passed two fluid, light yellow motions without any blood in either. The lung condition remained unchanged. His temperature was $100^{\circ}$ in the morning and $103^{\circ}$ in the evening. On the 21st the blood was again subjected to Widal's test and gave an incomplete reaction. His temperature was $99.6^{\circ}$ in the morning and $1028^{\circ}$ in the evening. On the $22 \mathrm{nd}$ his temperature was $99.4^{\circ}$ in the morning and $101 \cdot 6^{\circ}$ in the evening. He continued to do fairly well till the 23rd when he became very restless and had two large hæmorrhages from the bowel. A sweiling was noticed in the epigastrium; it was dull to percussion and no fuctuation was detected. The urine contained bile pigment. In the evening he had two large hæmorrhages and became collapsed; the pulse was fluttering and very weak and the extremities were cold, but he rallied under treatment. His temperature was $98^{\circ} 4^{\circ}$ in the morning and $100^{\circ}$ in the evening. On the 24th he had a number of hæmor rhages from the bowel and became again collapsed, but rallied under treatment by opium and champagne. His teroperature was $97^{\circ}$ in the morning and $96.4^{\circ}$ in the evening. On the 25th he passed a large number of small "tarry" motions involuntarily; the pulse became imperceptible and the respirations shallow and sighing, and he died quietly about $10 \mathrm{~A} . \mathrm{M}$. His temperature that morning was $96^{\circ}$.

Necropsy.-The right lung weighed 16 ounces; its substance was very anæmic. It was adherent at the base to the chest wall and diaphragm. A small portion of the base was consolidated, dark-red in colour, and leathery in consistence. The left lung weighed nine ounces and was very anæmic ; it was slightly adherent at the base. The heart weighed 10 ounces ; the walls of the right ventricle were thin, pale, and extensively infiltrated with fat. The liver was much enlarged, especially the left lobe, and weighed 84 ounces. It contained five abscesses, three in the right lobe and two in the left. Two of the abscesses were as large as turkey's eggs the others were smaller. One of the abscess cavities con tained bile, the others contained thin yeliow pus. The liver was adherent to the diaphragm. The spleen weighed eight ounces. The kidneys were very anæmic. The stomach and small intestine showed no abnormal appearances. Close to the ileo-cæcal valve and on it were a number of irregularly shaped ulcers, some of which were two-thirds of an inch long and others smaller. The mucous membrane of the two feet of the large intestine next the valve was congested and contained numerous ulcers, the largest being nearest the valve. Beyond a distance of six inches from the valve the ulcers were small, circular, and punched out in appearance. The mucous membrane of the remainder of the large intestine and rectum was quite healthy. There was no thickening of the walls of the bowel at any point. The mesenteric glands were swollen and soft.

The spleen was incised with a sterile knife and three tubes of Parietti's broth were inoculated from it. In all three tubes there grew a bacillus and a coccus, the bacteriological examination of which gave the following results. (a) The bacillus in Parietti broth produced a slight turbidity in 48 hours and a marked turbidity in 72 hours (at $90^{\circ} \mathrm{F}$.). In broth it produced turbidity in 24 hours; after a week's incubation at $90^{\circ} \mathrm{F}$. a pellicle formed. In gelatin-stab cultures there was a growth visible in 48 hours ; there was a greyish-white slow growth on the surface and a beaded growth in the substance of the gelatin. In gelatinstreak cultures there was growth visible in 48 hoursslow growth, bluish white, irregular edges; there was no liquefaction. In glucose-gelatin shake culture there was turbidity in 24 hours, with slight gas formation in from three to eight days. Sterilised milk did not coagulate in 10 days. On potato there was moist transparent growth in 24 hours (at $90^{\circ} \mathrm{F}$.) ; in a week the growth became thick and greyish. Agar streak culture gave a thick creamy-white growth. Gelatin plate culture gave a slow growth in 72 hours ; the colonies were granular and dark in the centre; there was no liquefaction. The microscopic characters of the organism were as follows: a short, thick, sluggishly motile bacillus with rounded ends. Stained by dilute carbolfuchsin some bacilli showed slightly stained central portions and deeply stained ends. Long th eat-like forms were also seen. (b) The coccus appeared to be streptococcus pyogenes from its cultivation characters.

The clinical characters of the case were at first puzzling, but latterly they were quite consistent with a diagnosis of enteric fever. Neither the clinical history nor the postmortem appearance supported a diagnosis of dysentery. I inspected the motions regularly and they never presented. any dysenteric characters. The bacillus isolated from the spleen resembled bacillus coli in most of its characters, but it did not coagulate milk, it only produced gas after from three to eight days' growth in glucose-gelatin, and at first the growth on potato resembled that of bacillus typhosus.

\section{TWO CASES OF PULSUS PARADOXUS.}

BY JOHN HAY, M.B. VICT.,

ASSISTANT MEDICAL OFFICER, MLLL-ROAD INFIRMARY, LIVERPOOL.

UNDER certain conditions the pulse becomes distinctly smaller during inspiration or completely disappears and this form of pulse is named the "pulsus paradoxus." It is not of common occurrence, though probably not so rare as is usually supposed, often being looked upnn simply as an irregularity of the pulse whilst the rhythmical character of the irregularity is not perceived. This condition is usually bilateral but is occasionally not so, an interesting case of this nature being recorded by Dr. Thomas Harris of Manchester in THE LANCET of April 22nd, 1899, p. 1072

As to the value of the sign, opinions differ greatly, but I think that we may take it that pulsus paradoxus is not of itself pathognomonic of any condition, though it cannot be denied that it does exist in perhaps its most marked form in certain cases of indurative anterior mediastino-pericarditis ; but there are other conditions in which it is sometimes present, such as pericarditis with effusion uncomplicated by mediastinitis, also in pleuritic effusion and in patients suffering from great inspiratory dyspnœa.

The following two cases illustrate to some extent (1) these. three conditions as causative agents; (2) the relief 